\title{
Fourier spectrum of radially periodic images
}

\author{
Isaac Amidror \\ Laboratoire de Systèmes Périphériques, Ecole Polytechnique Fédérale de Lausanne, 1015 Lausanne, Switzerland
}

Received March 15, 1996; revised manuscript received September 27, 1996; accepted October 16, 1996

\begin{abstract}
Although the spectrum of radially periodic images is often expressed in terms of finite or infinite series of Bessel functions, such expressions do not clearly reveal the exact impulsive structure of the spectrum. An alternative Fourier decomposition of radially periodic images, in terms of circular cosine functions, is presented, and its significant advantages are shown. It is shown that the Fourier transform of the circular cosine function, which can be expressed in terms of a half-order derivative of the impulse ring $\delta(r-f)$, plays a fundamental role in the spectra of radially periodic functions. Just as any symmetric periodic function $p(x)$ in the one-dimensional case can be represented by a sum of cosines with frequencies of $f=1 / T, 2 / T, \ldots$ [the Fourier series decomposition of $p(x)$ ], a radially periodic function in the two-dimensional case can be decomposed into a circular Fourier series, which is a sum of circular cosine functions with radial frequencies of $f$ $=1 / T, 2 / T, \ldots$. This result can also be formulated in terms of the spectral domain: Just as the Fourier transform of a one-dimensional periodic function consists of impulse pairs located at $f=n / T$ (the Fourier transforms of the cosines in the sum), the Fourier spectrum of a radially periodic function in the twodimensional case consists of half-order derivative impulse rings with radii $f=n / T$ (which are the Fourier transforms of the circular cosines in the sum). The significance of these results is discussed, and it is briefly shown how they can be extended into dimensions other than two. (C) 1997 Optical Society of America
\end{abstract} [S0740-3232(97)00504-8]

Key words: Fourier analysis, Fourier spectrum, radially periodic images, circular Fourier series, impulse rings, half-order derivatives.

\section{INTRODUCTION}

Radially periodic images and their Fourier spectra occur quite frequently in optics. Typical cases are a circular grating with a square-wave radial profile, or a series of concentric, equispaced ring impulses (representing narrow circular slits). Their Fourier transforms arise, for example, in connection with the Fraunhofer diffraction pattern generated by these circular structures. Although the spectrum of such radially periodic images is often expressed in terms of a finite or infinite series of Bessel functions, these expressions do not explicitly reveal the exact impulsive structure of the spectrum. In this paper I present an alternative approach for representing radially periodic functions and their spectra, which is based on circular cosine functions, and I show its significant advantages.

Section 2 starts with some of the fundamental observations that motivated the present research. In Section 3 I derive the Fourier spectrum of the circular cosine function, $\cos (2 \pi f r)$, and show that it can be expressed in terms of the half-order derivative of the impulse ring $\delta(q-f)$. In Section 4 I show how a radially periodic image can be decomposed into a circular Fourier series of circular cosine functions with radial frequencies of $f$ $=1 / T, 2 / T, \ldots$, and I explain the significance of this result in the image domain as well as in the spectral frequency domain. Finally, in Section 5 I briefly show how the results can be generalized into spaces with dimensions other than two.

\section{PRELIMINARY OBSERVATIONS}

A radially periodic image is a circularly symmetric image on the two-dimensional (2D) plane whose intensity profile along its radius is periodic. A radially periodic image can therefore be expressed in terms of the polar coordinates $(r, \theta)$ as a function $g(r)$, which is periodic in $r(r>0)$. Figure 1(a) shows an example of a radially periodic image: This is a circular binary grating, i.e., the function obtained by revolving the periodic binary square wave $p(x)$ of Fig. 2(a) about the vertical axis. The radial period of this radially periodic image is $T$, its radial frequency is $f=1 / T$, and its radial pulse width (or opening) is $\tau$.

The Fourier spectrum of this radially periodic image, as obtained by computer with the 2D discrete Fourier transform (DFT), is shown in Fig. 1(b). A careful examination of this spectrum and of its cross section (paying due attention not to be misled by DFT artifacts such as folding over and leakage ${ }^{1}$ ) shows that this spectrum consists of a series of concentric impulse rings that have a peculiar radial profile, reminiscent of a dipole. However, unlike the annular dipole $\delta^{\prime}(r-a)$, each of these rings has remarkably unequal positive and negative peaks, and moreover, each of the rings posesses a weak, continuous wake trailing off toward the center of the spectrum. Furthermore, the radii of these concentric rings are integer multiples of $f=1 / T$, and in spite of their wakes, their impulse amplitudes behave essentially like the impulse amplitudes in the one-dimensional (1D) spectrum of $p(x)$, the cross section through the center of the image $g(r)$. [In our example of a circular binary grating, $p(x)$ is a binary square wave with the same period $T$ and opening $\tau$, as shown in Fig. 2(a); compare its spectrum, shown in Fig. 2(b), with Fig. 1(c)].

It should be emphasized that this peculiar behavior of the spectrum is by no means a DFT artifact but a true characteristic feature of the spectrum of radially periodic 


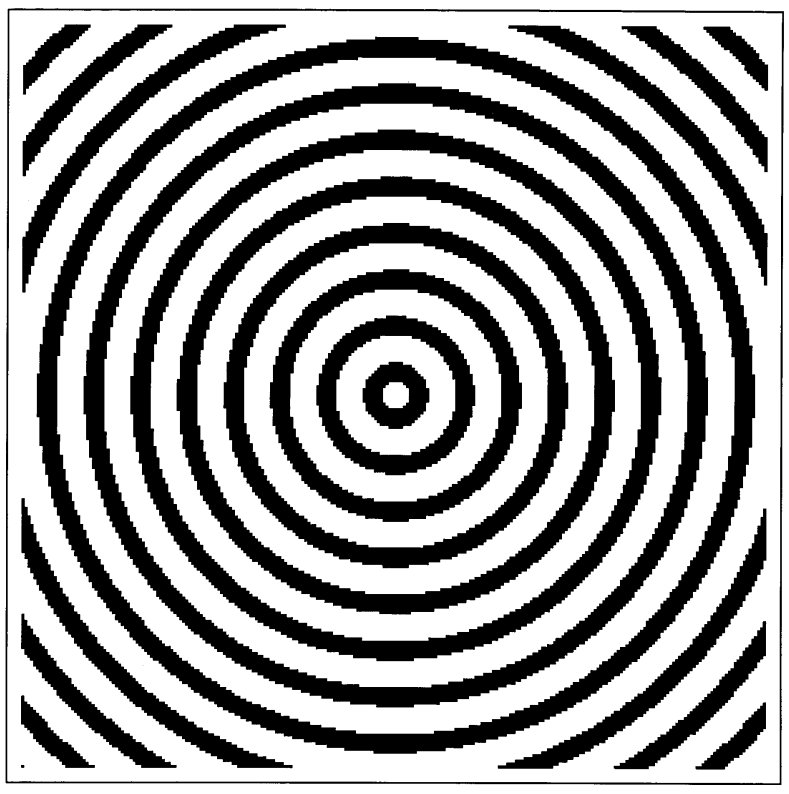

(a)

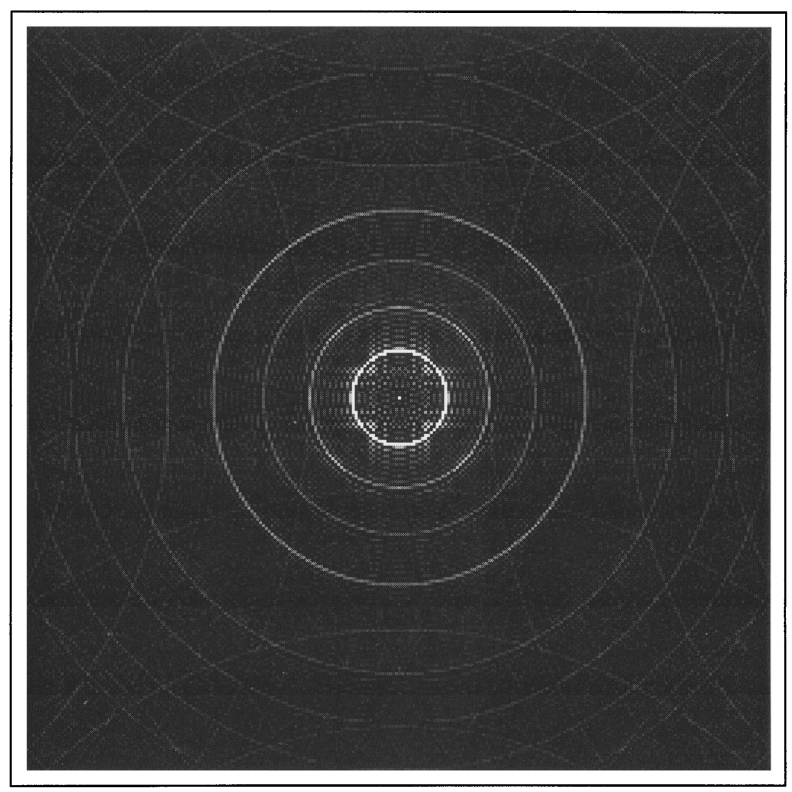

(b)

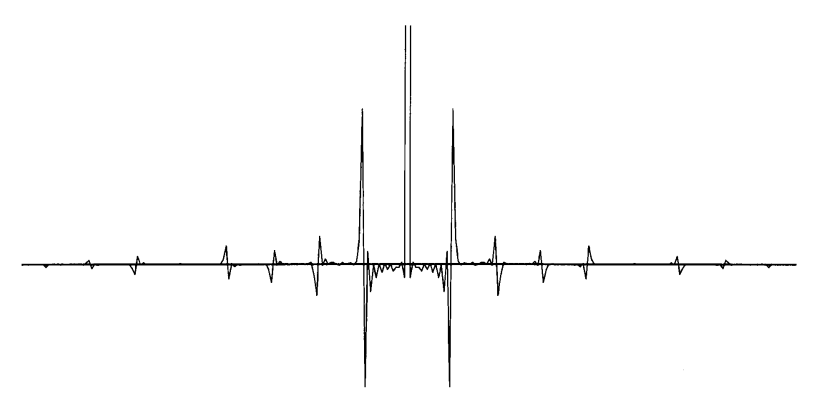

(c)

Fig. 1. (a) Circular binary grating with radial period $T=1$ and opening $\tau=3 / 5$. (b) Its Fourier spectrum as obtained by a 2D DFT (notice the folding-over artifacts that are due to the DFT). (c) Average cross section through the origin of this DFT (averaged through all directions $\theta=0^{\circ} \ldots 360^{\circ}$ to compensate for local DFT artifacts). images. This can be easily demonstrated in the case of our circular binary grating (Fig. 1), since in this case an analytic expression can be easily derived for the Fourier spectrum. The circular binary grating is expressed by

$$
\begin{aligned}
g(r)= & \operatorname{rect}\left(\frac{r}{\tau}\right)+\sum_{n=1}^{N}\left\{\operatorname{rect}\left[\frac{r}{2(n T+\tau / 2)}\right]\right. \\
& \left.-\operatorname{rect}\left[\frac{r}{2(n T-\tau / 2)}\right]\right\},
\end{aligned}
$$

(where $N \rightarrow \infty$ ), and its Fourier spectrum is immediately given $b^{2}$

$$
\begin{aligned}
G(q)= & \tau^{2} \operatorname{jinc}(\tau q)+\sum_{n=1}^{N}\left\{4\left(n T+\frac{\tau}{2}\right)^{2}\right. \\
& \times \operatorname{jinc}\left[2\left(n T+\frac{\tau}{2}\right) q\right]-4\left(n T-\frac{\tau}{2}\right)^{2} \\
& \left.\times \operatorname{jinc}\left[2\left(n T-\frac{\tau}{2}\right) q\right]\right\}
\end{aligned}
$$

where $\operatorname{jinc}(q)=J_{1}(\pi q) / 2 q$. By plotting Eq. (2) with more and more terms in the sum, i.e., with $N \rightarrow \infty$, one can see how it gradually approaches the characteristic behavior of the spectrum as shown in Figs. 1(b) and 1(c), namely, a series of concentric rings with the same particular properties as described above.

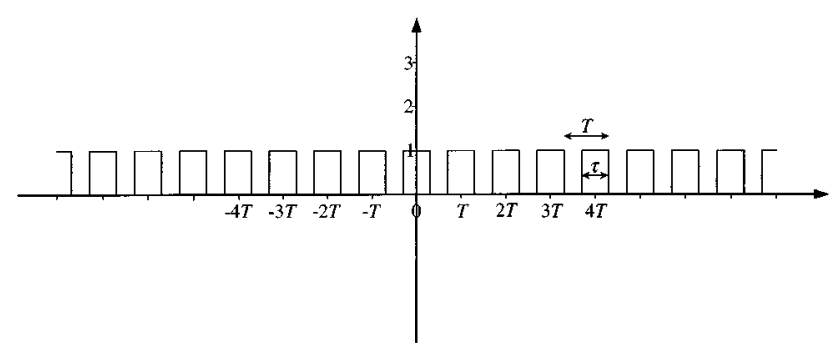

(a)

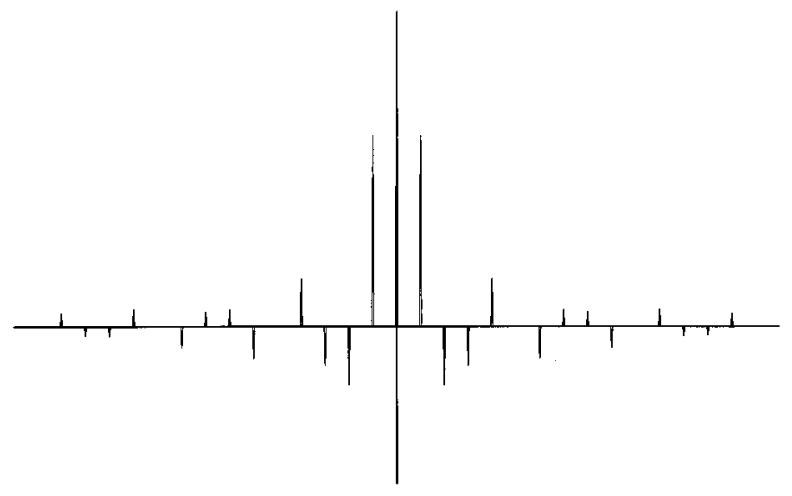

(b)

Fig. 2. (a) Cross section through the center of the circular binary grating of Fig. 1(a): This is a square wave with period $T$ $=1$ and opening $\tau=3 / 5$. (b) $1 \mathrm{D}$ spectrum of this binary square wave. Notice the agreement with Fig. 1(c) in the impulse signs and strengths; note in particular that every fifth impulse has a zero amplitude that is due to the opening ratio $\tau / T=3 / 5$ of the square wave, since the impulse amplitudes in the case of a square wave are given by $c_{n}=(1 / n \pi) \sin (n \pi \pi / T)$. 
This and other examples of radially periodic images and their spectra lead us to the following important observation: Although the spectrum of radially periodic images, like all circularly symmetric images, is often expressible in a natural way as a finite or infinite sum of Bessel functions, ${ }^{3,4}$ the individual terms of this sum do not explicitly correspond to the individual impulse rings of the spectrum and do not express their peculiar profile shapes. This suggests that the spectrum of radially periodic images can also be expressed in an alternative way, as a series of terms where the $n$th term explicitly represents the $n$th impulse ring. ${ }^{5}$

On the basis of our experience with periodic functions, it can be expected that each of these impulse rings should correspond to the spectrum of a circular cosine function [a radially periodic function with cosinusoidal radial profile; see Fig. 3(a)] given by

$$
g(x, y)=\cos \left(2 \pi f \sqrt{x^{2}+y^{2}}\right)
$$

or in polar coordinates:

$$
g(r)=\cos (2 \pi f r),
$$

where $f=1 / T, 2 / T, \ldots$. If this hypothesis is confirmed, one would be able to decompose any radially periodic image into a circular Fourier series, which is a sum of circular cosine functions with radial frequencies of $f$ $=1 / T, 2 / T, \ldots$, just as any $1 \mathrm{D}$ periodic function is decomposed into a sum of cosines. To this end, it is therefore necessary to find the Fourier transform of circular cosine (3) [or the Hankel transform of Eq. (4). The Hankel transform is an equivalent way to express the 2D Fourier transform of a circularly symmetric function, based on its radius; ${ }^{6}$ here both terms will be used interchangeably, depending on the coordinate system implied, polar or Cartesian.]

\section{SPECTRUM OF THE CIRCULAR COSINE}

In spite of its simple appearance, the circular cosine function cannot be easily found in standard tables of Fourier transform pairs. In fact, the closest hint one can find in literature is hidden in the following general Fourier (or rather Hankel) transform pair ${ }^{7}$ :

$$
\frac{1}{r^{\mu}} J_{\mu}(2 \pi f r) \stackrel{\mathscr{C}}{\leftrightarrow} \frac{\pi^{\mu-1}}{f^{\mu} \Gamma(\mu)}\left(f^{2}-q^{2}\right)^{\mu-1} \operatorname{rect}\left(\frac{q}{2 f}\right),
$$

where $\operatorname{rect}(q / 2 f)$ means truncation to zero beyond the circle of radius $f$, and

$$
r=\sqrt{x^{2}+y^{2}}, \quad q=\sqrt{u^{2}+v^{2}} .
$$

This general formula gives several interesting Hankel transform pairs for various values of $\mu$. In particular, for $\mu=-1 / 2$ it gives the Hankel transform of circular cosine (4), since

$$
\cos r=\sqrt{\pi r / 2} J_{-1 / 2}(r) .
$$

It should be noted that transform pair (5) is usually given in the literature only for $\mu>0$ [probably because for $\mu \leqslant 0$ the functions on the left-hand side of Eq. (5) do not properly decay, and consequently their Hankel transforms include a "wild" (impulsive) behavior on the border

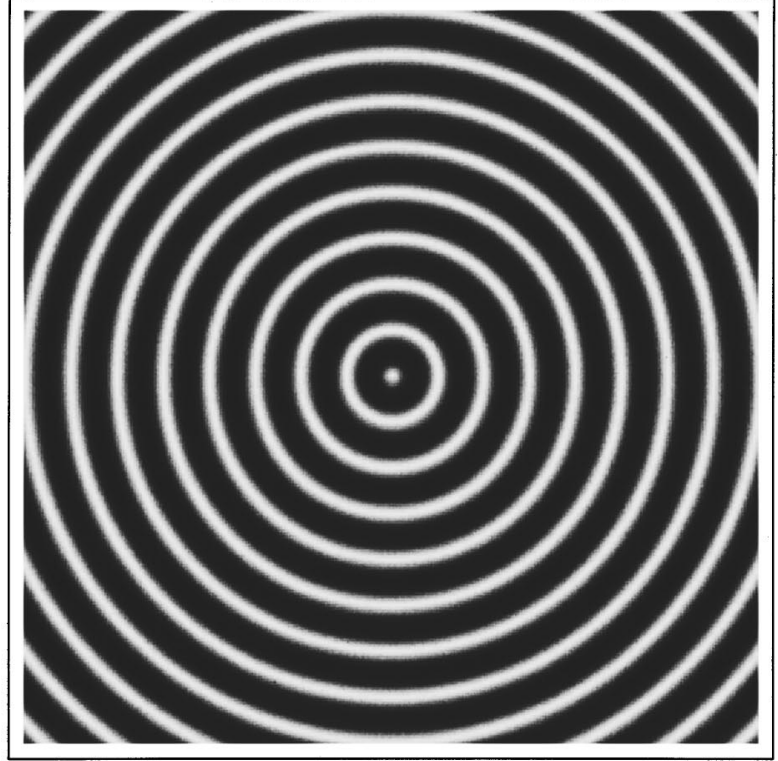

(a)

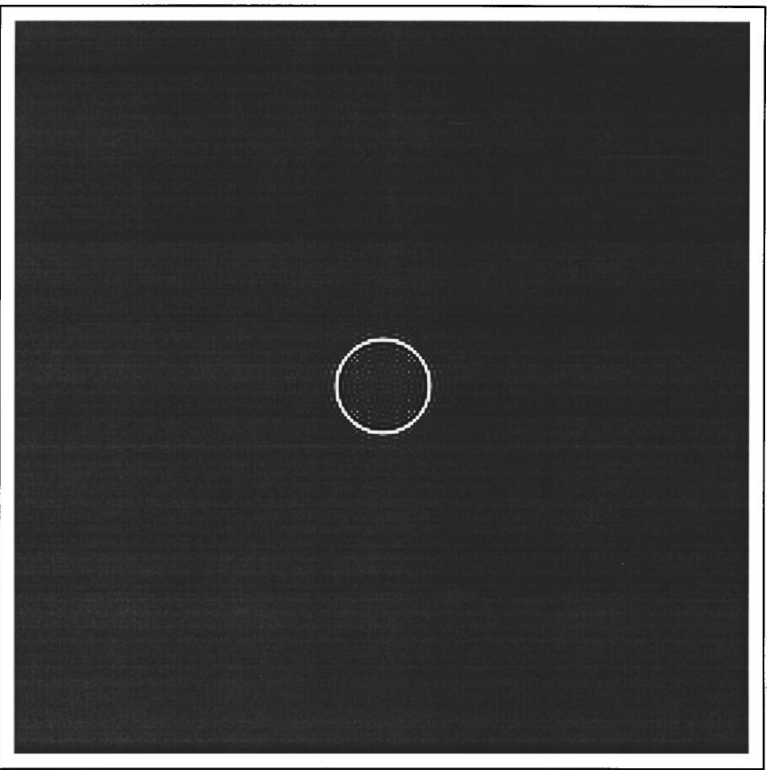

(b)

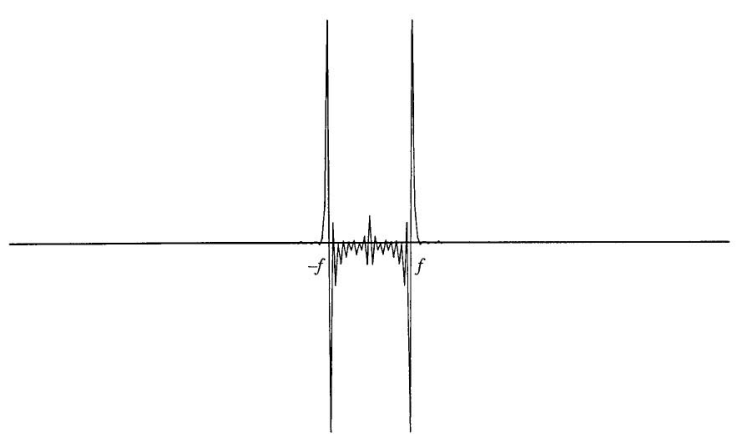

(c)

Fig. 3. (a) Circular cosine function $\cos (2 \pi f r)$. (b) Its Fourier spectrum as obtained by a 2D DFT (notice the leakage artifacts: each point of the ring is convolved with the Fourier transform of the truncation window). (c) Average cross section through the origin of this DFT (averaged through all directions $\theta=0^{\circ} \ldots 360^{\circ}$ to compensate for local DFT artifacts). 
of their circular spectrum support]. However, according to Ref. 8, formula (5) is also valid for noninteger negative values of $\mu$, including our case of $\mu=-1 / 2$. For $\mu$ $=-1 / 2$, formula $(5)$ therefore gives

$$
\cos (2 \pi f r) \stackrel{\mathscr{H}}{\leftrightarrow}-\frac{f}{2 \pi} \frac{1}{\left(f^{2}-q^{2}\right)^{3 / 2}} \operatorname{rect}\left(\frac{q}{2 f}\right) .
$$

The Fourier spectrum of circular cosine (3), as obtained by the 2D DFT [see Figs. 3(b) and 3(c)], confirms this result for the interior of the ring, $\{q<f\}$. However, it also indicates that the behavior of the spectrum on its singular support, $\{q=f\}$, is more complicated: In addition to the negative peak at the internal side of the ring, as predicted by relation (7), it clearly also shows a positive impulsive behavior at the external side of the ring, so that a vertical section through the spectrum origin would look like Fig. 4.

Note that the external impulsive border of this ring is sharp, whereas the internal, negative peak of the ring is characterized by a smooth decay transition in the form of a continuous wake. As in the case of Fig. 1, it could be argued that this peculiar impulsive behavior represents an artifact due to the limitations of the DFT in representing the nondecaying circular cosine function [Eq. (3)]; however, as will be shown below, a deeper investigation shows that this behavior indeed represents an inherent feature of this Fourier transform.

To this end, we need to make a short digression and to recall the notion of a half-order derivative. Although not largely widespread, the notion of fractional-order derivatives and integrals is not new in mathematics. Although the original definition of the derivative of a function is given only for derivatives of integer orders, it is well known that this definition can be extended to noninteger orders by using an elementary property of Fourier transforms. According to the derivative theorem, ${ }^{9}$ if the Fourier transform of $f(x)$ is $F(u)$, then the Fourier transform of $f^{\prime}(x)$ is $i 2 \pi u F(u)$; and hence, if we apply the theorem in succession, it follows that for any integer $k$ the Fourier transform of $f^{(k)}(x)$ is $(i 2 \pi u)^{k} F(u)$. But since $(i 2 \pi u)^{\lambda} F(u)$ is meaningful for noninteger values of $\lambda$ as well, it can be regarded as the definition of $f^{(\lambda)}(x)$ for any real $\lambda$. In particular, for $\lambda=1 / 2$ we obtain

$$
f^{(1 / 2)}(x)=\mathscr{F}^{-1}\left[(i 2 \pi u)^{1 / 2} F(u)\right] .
$$

Note that this definition is consistent with the usual definition of $f^{\prime}(x)$ since its application twice in succession results in the first-order derivative of $f(x)$ : $\left[f^{(1 / 2)}(x)\right]^{(1 / 2)}$ transforms into $(i 2 \pi u)^{(1 / 2)}(i 2 \pi u)^{(1 / 2)} F(u)$ $=i 2 \pi u F(u)$, which is indeed the Fourier transform of $f^{\prime}(x)$. The various properties of fractional-order derivatives as well as alternative ways of defining them can be found in specialized books on the subject. ${ }^{10,11}$

Now, it is well known that integer-order derivatives can be defined for generalized functions such as the Dirac impulse $\delta(x)$, giving the dipole $\delta^{\prime}(x)$, the quadrupole $\delta^{(2)}(x)$, etc. ${ }^{12}$ It is, however, less widely known that fractional-order derivatives can also be defined for generalized functions such as $\delta(x)$. An explicit expression for the fractional-order derivatives of $\delta(x)$ can be found in advanced books on generalized functions such as Ref. 13:

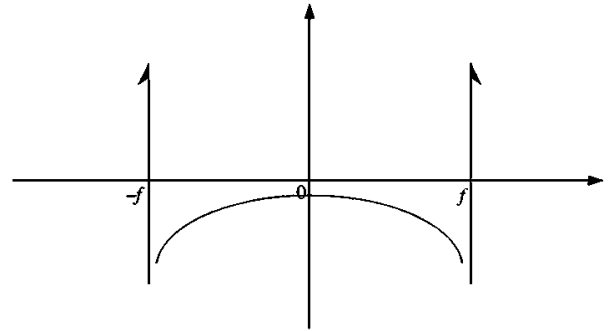

Fig. 4. Schematic plot of a cross section through the spectrum of circular cosine function $\cos (2 \pi f r)$. Notice the positive impulsive peak on the external border of the ring.

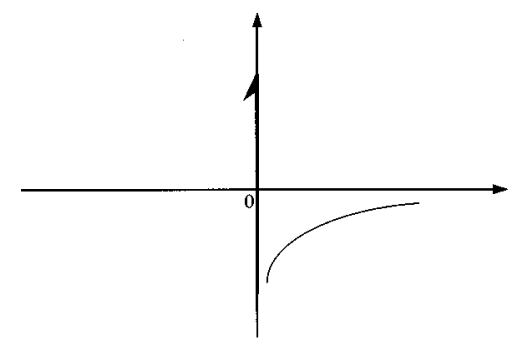

Fig. 5. Schematic plot of $\delta^{1 / 2}(x)$, the half-order derivative of the impulse $\delta(x)$.

$$
\delta^{(\lambda)}(x)=\frac{1}{\Gamma(-\lambda)} \frac{1}{x^{\lambda+1}} \operatorname{step}(x),
$$

where $\operatorname{step}(x)$ is defined as 0 for $x<0$ and 1 for $x>0$; in particular, this gives for $\lambda=1 / 2$,

$$
\delta^{(1 / 2)}(x)=-\frac{1}{2 \sqrt{\pi}} \frac{1}{x^{3 / 2}} \operatorname{step}(x) .
$$

As is shown in Appendix B, Eq. (9) describes the properties of $\delta^{(1 / 2)}(x)$ to the right of $x=0$; but when we approach $\delta^{(1 / 2)}(x)$ by a sequence of functions that are defined on both sides of $x=0$, it becomes apparent that $\delta^{(1 / 2)}(x)$ has at the point $x=0$ an impulsive behavior: It has a positive impulsive peak on the left-hand side of $x=0$, while on the right-hand side of $x=0$ it has a negative peak, which smoothly decays in the form of a negative continuous wake trailing off asymptotically in the positive direction of the $x$ axis (see Fig. 5). Other properties of $\delta^{(1 / 2)}(x)$ are given in Appendix B.

Now, from Eq. (9) we obtain

$$
\delta^{(1 / 2)}\left(f^{2}-q^{2}\right)=-\frac{1}{2 \sqrt{\pi}} \frac{1}{\left(f^{2}-q^{2}\right)^{3 / 2}} \operatorname{rect}\left(\frac{q}{2 f}\right)
$$

[note the truncation beyond the radius $f$, due to the inside-out inversion of $\delta^{(1 / 2)}\left(f^{2}-q^{2}\right)$ with respect to $\left.\delta^{(1 / 2)}\left(q^{2}-f^{2}\right)\right]$. A comparison of Eqs. (7) and (10) (see also Figs. 4 and 5) makes it clear that the spectrum (or the Hankel transform) of the circular cosine function can be expressed in terms of $\delta^{(1 / 2)}()$ as follows, thus emphasizing its impulsive behavior:

$$
\cos (2 \pi f r) \stackrel{\mathscr{H}}{\leftrightarrow} \frac{f}{\sqrt{\pi}} \delta^{(1 / 2)}\left(f^{2}-q^{2}\right) .
$$

This expression can be further simplified by expressing $\delta^{(1 / 2)}\left(f^{2}-q^{2}\right)$ in terms of the half-order derivative of the 
simple impulse ring, $\delta^{(1 / 2)}(f-q)$ : It is known that for any integer $k$ there exists the relation ${ }^{14}$

$$
\delta^{(k)}\left(r^{2}-c^{2}\right)=\frac{1}{(r+c)^{k+1}} \delta^{(k)}(r-c) .
$$

However, this relation turns out to be valid also for noninteger values of $k$, denoted below by $\lambda$, since by Eq. (8) we have (for $r, c>0$ ),

$$
\begin{aligned}
\delta^{(\lambda)}\left(r^{2}-c^{2}\right)= & \frac{1}{\Gamma(-\lambda)} \frac{1}{\left(r^{2}-c^{2}\right)^{\lambda+1}} \operatorname{step}\left(r^{2}-c^{2}\right) \\
= & \frac{1}{\Gamma(-\lambda)} \frac{1}{(r+c)^{\lambda+1}(r-c)^{\lambda+1}} \\
& \times \operatorname{step}(r-c) \\
= & \frac{1}{(r+c)^{\lambda+1}} \delta^{(\lambda)}(r-c) .
\end{aligned}
$$

Therefore we obtain the following expression for the spectrum of the circular cosine with $f>0$ :

$$
\cos (2 \pi f r) \stackrel{\mathscr{B}}{\leftrightarrow} \frac{f}{\sqrt{\pi}} \frac{1}{(f+q)^{3 / 2}} \delta^{(1 / 2)}(f-q) .
$$

In the case of $f=0$, in which the cosine becomes identical 1 , the spectrum is given by the well-known transform pair $^{15}$

$$
1 \stackrel{\mathscr{H}}{\leftrightarrow} \frac{1}{\pi|q|} \delta(q) .
$$

Equation (12) indeed confirms observations with the 2D DFT that clearly show that as $f$ increases, not only does the radius of the impulse ring increase, but also its wake becomes weaker. Note that $\delta^{(1 / 2)}(f-q)$ is the inside-out inverted counterpart of the $\delta^{(1 / 2)}(q-f)$ ring, where the negative wake trails off inward, toward the center, and the positive impulsive peak is located in the outer side (see Fig. 4).

\section{REPRESENTATION OF THE SPECTRUM AS A SERIES OF IMPULSE RINGS}

The scheme for disentangling one impulse ring from the other rings in the spectrum of a radially periodic image (in spite of their overlapping wakes) has been proposed in a paper by Bracewell and Thompson. ${ }^{16}$ In that paper the image in question was $g(r)=\Sigma_{n=1}^{N} \delta(r-n T)$, whose spectrum (Hankel transform) is $G(q)$ $=\sum_{n=1}^{N} 2 \pi n T J_{0}(2 \pi n T q)$. In our present discussion we shall follow a similar approach, applying it to a general, arbitrary radially periodic image $g(r)$.

We start with the following definition: Let $f(x)$ be a 1D function defining a surface over the $(x, y)$ plane. The circular function $s(r)$ is said to be the spin average of $f(x)$, denoted by $s(r)=\mathscr{S}[f(x)]$, if

$$
s(r)=\frac{1}{2 \pi} \int_{0}^{2 \pi} f(r \cos \theta) \mathrm{d} \theta .
$$

This means that $s(r)$ is the circular surface obtained by averaging the height of the surface of $f(x)$ over a circle of radius $r$ about the origin in the $(x, y)$ plane. Various properties of the spin-average operation are discussed in Refs. 16 and 17. In particular, it is shown that this operation is linear and has a unique inverse; a table of various functions and their spin averages is given in Ref. 17.

Now let $g(r)$ be a radially periodic image, and let $G(q)$ be its Hankel transform. The fourth theorem concerning the spin average given in Ref. 18 says that if

$$
G(q)=\mathscr{S}[H(u)],
$$

then

$$
\pi|r| \mathscr{H}[G(q)]=\mathscr{F}[H(u)],
$$

where $\mathscr{H}[G(q)]$ is the Hankel transform of $G(q)$, namely, $g(r)$, and $\mathscr{F}[H(u)]$ is the 1D Fourier transform of $H(u)$. If we denote by $h(x)$ the function whose Fourier transform is $H(u)$, we can reformulate Eq. (15) as

$$
\pi|r| g(r)=\mathscr{F}\{\mathscr{F}[h(x)]\},
$$

and since $\mathscr{F}\{\mathscr{F}[h(x)]\}=h(-x)$ we obtain, remembering that $h(x)$ is an even function, and concentrating our attention on the $x$ axis,

$$
h(x)=\pi|x| p(x),
$$

where $p(x)$ is the periodic cross section of the radially periodic image $g(r)$.

Now let $P(u)=\Sigma_{n=-\infty}^{\infty} c_{n} \delta(u-n / T)$ be the spectrum of the periodic cross section $p(x)$, where $c_{n}$ are its impulse amplitudes [i.e., the Fourier series coefficients of $p(x)$ ]. Using the convolution theorem, we obtain from Eq. (16),

$$
H(u)=F(u) * \sum_{n=-\infty}^{\infty} c_{n} \delta\left(u-\frac{n}{T}\right)
$$

where $F(u)$ is the $1 \mathrm{D}$ Fourier transform of $f(x)=\pi|x|$. (This Fourier transform can be found, for example, in Ref. 19 , but as we will see below, its explicit form is not really needed here.) We have, therefore,

$$
\begin{aligned}
H(u)= & c_{0} F(u)+\left[c_{-1} F\left(u+\frac{1}{T}\right)+c_{1} F\left(u-\frac{1}{T}\right)\right] \\
& +\left[c_{-2} F\left(u+\frac{2}{T}\right)+c_{2} F\left(u-\frac{2}{T}\right)\right]+\ldots .
\end{aligned}
$$

But since $p(x)$ is symmetric, we have $c_{-n}=c_{n}$, so that

$$
\begin{array}{r}
H(u)=c_{0} F(u)+c_{1}\left[F\left(u+\frac{1}{T}\right)+F\left(u-\frac{1}{T}\right)\right] \\
+c_{2}\left[F\left(u+\frac{2}{T}\right)+F\left(u-\frac{2}{T}\right)\right]+\ldots .
\end{array}
$$

Remembering now that $H(u)$ spin averages into the spectrum $G(q)$, let us denote

$$
\begin{aligned}
& G_{0}(q)=\mathscr{S}[F(u)], \\
& G_{n}(q)=\mathscr{S}\left\{c_{n}\left[F\left(u+\frac{n}{T}\right)+F\left(u-\frac{n}{T}\right)\right]\right\} .
\end{aligned}
$$

Therefore, by taking the spin average of both sides of Eq. (17), we obtain a new decomposition of the spectrum $G(q)$, as follows: 


$$
G(q)=c_{0} G_{0}(q)+c_{1} G_{1}(q)+c_{2} G_{2}(q)+\ldots .
$$

Now, again using the same theorem concerning the spin average [see Eqs. (14) and (15) above], we find that the Hankel transforms of $G_{0}(q)$ and of $G_{n}(q)$ are given by

$$
\begin{aligned}
& g_{0}(r)=\frac{1}{\pi|r|} \mathscr{F}[F(u)], \\
& g_{n}(r)=\frac{1}{\pi|r|} \mathscr{F}\left[F\left(u+\frac{n}{T}\right)+F\left(u-\frac{n}{T}\right)\right] .
\end{aligned}
$$

Remembering that $F(u)$ is the $1 \mathrm{D}$ Fourier transform of $f(r)=\pi|r|$, we obtain

$$
g_{0}(r)=\frac{1}{\pi|r|} f(-r)=\frac{1}{\pi|r|} \pi|r|=1,
$$

and by the modulation theorem, ${ }^{9}$

$$
\begin{aligned}
g_{n}(r) & =\frac{1}{\pi|r|} \mathscr{F}\left\{\mathscr{F}\left[2 f(r) \cos \left(2 \pi \frac{n}{T} r\right)\right]\right\} \\
& =\frac{1}{\pi|r|} 2 f(-r) \cos \left(2 \pi \frac{n}{T} r\right) \\
& =\frac{1}{\pi|r|} 2 \pi|r| \cos \left(2 \pi \frac{n}{T} r\right)=2 \cos \left(2 \pi \frac{n}{T} r\right) .
\end{aligned}
$$

In other words, $g_{n}(r)$, the Hankel transform of $G_{n}(q)$, is simply a circular cosine with radial frequency of $n / T$ and amplitude of 2 .

We see, therefore, that if we take a Hankel transform of Eq. (20), our original radially periodic image $g(r)$ can be expressed in the form

$$
\begin{aligned}
g(r) & =c_{0} g_{0}(r)+c_{1} g_{1}(r)+c_{2} g_{2}(r)+\ldots \\
& =c_{0}+2 \sum_{n=1}^{\infty} c_{n} \cos \left(2 \pi \frac{n}{T} r\right)
\end{aligned}
$$

where $c_{n}$ are the same Fourier series coefficients (impulse amplitudes) as in $p(x)$, the periodic cross section of $g(r)$. Note that the factor 2 appears here as a result of the use of a one-sided summation rather than the two-sided summation usually used in the 1D case: $p(x)$ $=\sum_{n=-\infty}^{\infty} c_{n} \cos (2 \pi x n / T)$.

Returning now to the spectral domain, we can find the spectra of Eqs. (21) and (22) by using the Hankel transform of the identical-1 function ${ }^{15}$ and the Hankel transform given by Eq. (12),

$$
\begin{aligned}
& G_{0}(q)=\frac{1}{\pi|q|} \delta(q), \\
& G_{n}(q)=\frac{2}{\sqrt{\pi}} \frac{n / T}{(n / T+q)^{3 / 2}} \delta^{(1 / 2)}\left(\frac{n}{T}-q\right),
\end{aligned}
$$

and therefore the explicit form of Eq. (20), the spectrum of the radially periodic function $g(r)$, is

$$
\begin{aligned}
G(q)= & c_{0} \frac{1}{\pi|q|} \delta(q)+\frac{2}{\sqrt{\pi}} \sum_{n=1}^{\infty} c_{n} \frac{n / T}{(n / T+q)^{3 / 2}} \\
& \times \delta^{(1 / 2)}\left(\frac{n}{T}-q\right) .
\end{aligned}
$$

Let us stop now for a moment to discuss the results that we have just obtained.

As we can see, Eq. (23) is a decomposition of the radially periodic function $g(r)$ into a circular Fourier series that is a sum of circular cosine functions with radial frequencies of $f=1 / T, 2 / T, \ldots$. This decomposition of $g(r)$ is closely related to the decomposition of its periodic cross section $p(x)$ into a 1D Fourier series: The coefficients of the circular Fourier series decomposition of $g(r)$ are the same as in the 1D Fourier series development of $p(x)$.

Turning now to the spectral domain, we see that expression (24), the spectrum of (23), is exactly the alternative expression that we were seeking for the spectrum of a radially periodic function $g(r)$. As we can see, this is a sum of terms $G_{n}(q)$, each of which is the spectrum (Hankel transform) of the circular cosine function $g_{n}(r)$ with radial frequency of $n / T$. As we already know from Section 3 [Eq. (12)], the spectrum of the circular cosine $\cos (2 \pi r n / T)$ is an impulse ring of radius $n / T$, with a negative wake trailing off toward the spectrum center and which can be expressed in terms of a half-order derivative of the impulse ring $\delta(q-n / T)$. We see therefore that $G_{n}(q)$ represents, indeed, the $n$th impulse ring in the spectrum $G(q)$ of our radially periodic image $g_{n}(r)$. We thus obtained the following remarkable result: The spectrum $G(q)$ of a radially periodic image $g(r)$ consists of a series of impulse rings $G_{n}(q)$, whose radii are $n / T$ and whose strengths are (up to a scaling factor) $c_{n}$, where $T$ is the radial period of our given image $g(r)$ and $c_{n}$ are the Fourier series coefficients of its $1 \mathrm{D}$ cross section $p(x)$.

We see, therefore, that just as the spectrum of any 1D periodic function $p(x)$ consists of impulse pairs located at the frequencies $f= \pm n / T$, the spectrum of a radially periodic function $g(r)$ in the $2 \mathrm{D}$ case is composed of concentric impulse rings of radii $f=n / T$. However, these rings have a particular, unusual shape: Each of the rings has a peculiar impulsive behavior, resembling that of a dipole, on the perimeter of a circle with radius of $f=n / T$; but unlike the impulse ring $\delta(q-n / T)$ it does not represent a pure radial frequency component of $f=n / T$, since it also includes lower radial frequencies (albeit of negligible amplitudes) that are due to its wake, which trails off toward the spectrum center.

These results fully confirm our hypothesis of Section 2, which was based there only on experimental observations through the 2D DFT of radially periodic images.

\section{ONE-, TWO-, AND THREE-DIMENSIONAL CASES}

Although our main interest in the present paper is in radially periodic images in the $2 \mathrm{D}$ case, it will be interesting to see how our results can be extended into cases of other dimensions. In this section I will explain very briefly (and without proofs) the situation in the one- and three- 
dimensional cases, and I will show the relationship between the $1 \mathrm{D}, 2 \mathrm{D}$, and $3 \mathrm{D}$ cases, thus opening the way to a generalization to the $n$-dimensional case.

The $1 \mathrm{D}$ case is obviously the simplest. A radially periodic image in the $1 \mathrm{D}$ case is simply a symmetric, periodic function of one variable, which can be denoted by $g(|x|)$. In this case our function can be decomposed into a sum of cosine functions, which is the classical, wellknown Fourier series decomposition. In the spectral domain, the $n$th cosine of the Fourier series corresponds to a $1 \mathrm{D}$ impulse ring, which is simply an impulse pair, $\delta(u$ $+n / T)$ and $\delta(u-n / T)$.

In the $2 \mathrm{D}$ case, as we have just seen in Section 4 , a radially periodic image can be decomposed into a circular Fourier series, i.e., a sum of circular cosine functions. In terms of the spectral domain, the $n$th circular cosine represents the $n$th impulse ring in the spectrum, each of these rings being essentially a half-order derivative of the simple impulse ring $\delta(q-n / T)$. (Note the inside-out inversion of $\delta^{(1 / 2)}(n / T-q)$, owing to which the wake is trailing inward!)

A similar result can be also obtained for the 3D case. In this case the spherically periodic image $g(r)$ can be decomposed into a spherical Fourier series, which is a sum of spherical cosines. This corresponds in the spectral domain to a series of impulsive spheres, each of which is the $3 \mathrm{D}$ equivalent of the Hankel transform ${ }^{20}$ of the spherical cosine function $\cos (2 \pi r n / T)$. This Hankel transform can be expressed, this time, in terms of the first-order derivative of the impulsive sphere $\delta(q-n / T)$.

As we can see, the underlying concept is the same for all dimensions-but each increment of 1 in the space dimension $n$ corresponds to an increment of $1 / 2$ in the order of the derivative in the concentric rings (or spheres) that constitute the corresponding $n$-dimensional spectrum. It is interesting that in odd dimensions the impulse rings are really purely impulsive, whereas in even dimensions the fractional-order derivative implies the existence, in each of the impulse rings, of a trailing wake that gradually decreases toward the center of the spectrum.

\section{CONCLUSIONS}

The main result presented in this paper is that a radially periodic function $g(r)$ can be decomposed into a circular Fourier series, which is a sum of circular cosine functions with radial frequencies of $f=1 / T, 2 / T, \ldots$. It is shown that this decomposition of $g(r)$ is closely related to the decomposition of its periodic cross section $p(x)$ into a 1D Fourier series: The coefficients $c_{n}$ of the circular Fourier series decomposition of $g(r)$ are the same as the coefficients in the Fourier series development of $p(x)$.

In terms of the spectral domain, the spectrum of a radially periodic function $g(r)$ consists of a dc impulse plus a series of impulse rings with radii of $f=1 / T, 2 / T, \ldots$, each of which is the spectral representation of a corresponding circular cosine function in the image domain decomposition. It is shown that these impulse rings have some peculiar properties, notably a wake that trails off toward the spectrum center, and that each of the rings can be expressed in terms of a half-order derivative of the simple impulse ring $\delta(r-f)$. Finally, I also indicate how these considerations can be extended into the general $n$-dimensional case.

The most significant advantage of this decomposition of $g(r)$ over other possible decompositions, such as the development into a series of Bessel functions, is that our decomposition explicitly reveals the impulse ring structure of the spectrum and the special properties of each of these rings: in our decomposition, the $n$th term explicitly represents the $n$th impulse ring in the spectrum of $g(r)$.

The phenomenon of circular impulse rings having a wake trailing off behind them in a $2 \mathrm{D}$ space is well known in various fields of optics and physics (for example, $\mathrm{cf}$. the wave propagation in 1,2 , and 3 dimensions ${ }^{21,22}$ ). However, the possible connection of such physical phenomena with fractional-order derivatives such as the half-order derivative of an impulse ring have rarely been realized in the literature (with only a few exceptions, such as in the book by Lighthill ${ }^{23}$ in the context of sound wave propagation or in the above-cited paper by Bracewell and Thompson). ${ }^{16}$ I believe that expressing such phenomena in terms of fractional impulse derivatives may often open the way to significant simplifications in handling such expressions, much like the simplifications obtained by using the notion of the simple impulse $\delta(x)$-provided that the properties of fractional-order impulse derivatives are well understood and correctly used (see Appendix B).

\section{APPENDIX A: COMPLETENESS AND ORTHOGONALITY CONSIDERATIONS}

In this appendix I briefly discuss the completeness and orthogonality properties of the decomposition of radially periodic images into circular Fourier series. In this introductory discussion I follow the same main lines as in the book by Gaskill. ${ }^{24}$

It is often desirable to decompose a given function $g(x)$ into a linear combination of a set of more elementary functions $\left\{f_{n}(x)\right\}, n=1,2,3, \ldots$ called basis functions:

$$
g(x)=\sum_{n} c_{n} f_{n}(x)
$$

The motivation is that doing so greatly simplifies certain mathematical operations, and, moreover, such a decomposition often allows a much deeper insight to be gained concerning the nature of the problem in question.

Many different sets of basis functions $\left\{f_{n}(x)\right\}$ can be used for this purpose, but if the set we have chosen is endowed with two important properties, namely, completeness and orthogonality, we are in a particularly lucky situation.

A set of functions $\left\{f_{n}(x)\right\}$ is said to be orthogonal (on a certain interval $T$ ) if for any $m$ and $n$ we have

$$
\int_{T} f_{m}(x) f_{n}(x) \mathrm{d} x=\left\{\begin{array}{ll}
0 & \text { if } m \neq n \\
A \neq 0 & \text { if } m=n
\end{array} .\right.
$$

The set of functions $\left\{f_{n}(x)\right\}$ is said to be complete if there exists no function $h(x)$ on the interval $T$, other than the identical zero function, such that for all members $f_{n}(x)$ of the set we have 


$$
\int_{T} h(x) f_{n}(x) \mathrm{d} x=0 .
$$

As shown by Gaskill ${ }^{24}$ and in other references, the orthogonality condition on the basis functions $\left\{f_{n}(x)\right\}$ allows us to determine easily the coefficients $c_{n}$ of decomposition (A1) independently of one another:

$$
c_{n}=\frac{1}{A} \int_{T} g(x) f_{n}(x) \mathrm{d} x .
$$

The completeness condition, on its part, ensures us that any nonzero square-integrable function $g(x)$ can be decomposed into a linear combination of the functions $\left\{f_{n}(x)\right\}$ as in Eq. (A1).

The most well-known examples of complete and orthogonal sets of basis functions $\left\{f_{n}(x)\right\}$ are

$$
\begin{array}{ll}
\text { (1) } \exp (i 2 \pi n f x), & n \in \mathbb{Z}, \\
\text { (2) } \sin (2 \pi n f x), & n \in \mathbb{Z}, \\
\text { (3) } \cos (2 \pi n f x), & n \in \mathbb{Z} .
\end{array}
$$

The third case is exactly the set of basic functions that is used in the $1 \mathrm{D}$ decomposition of a symmetric, periodic function into a Fourier series:

$$
p(x)=\sum_{n=-\infty}^{\infty} c_{n} \cos \left(2 \pi \frac{n}{T} x\right),
$$

with coefficients

$$
c_{n}=\frac{1}{T} \int_{T} p(x) \cos \left(2 \pi \frac{n}{T} x\right) \mathrm{d} x .
$$

The orthogonality of the basis functions $\{\cos (2 \pi x n / T)\}$ simply follows from the fact that

$$
\int_{T} \cos \left(2 \pi \frac{m}{T} x\right) \cos \left(2 \pi \frac{n}{T} x\right) \mathrm{d} x=\left\{\begin{array}{cc}
0 & \text { if } m \neq n \\
T / 2 & \text { if } m=n
\end{array} .\right.
$$

The case of $m \neq n$ follows from the identity $\cos \alpha \cos \beta=\frac{1}{2} \cos (\alpha+\beta)+\frac{1}{2} \cos (\alpha-\beta)$, by rewriting of the cosine product as a sum of two cosines. Since the frequencies of these cosines are integer multiples of $1 / T$, each of them has on any full-period interval $T$ as much negative area as positive area, and thus the total area is zero.

Let us now return to the case of interest, the decomposition of a radially periodic image $g(r)$ into a circular Fourier series [Eq. (23)]. The completeness of the set $\{\cos (2 \pi r n / T)\}$ in this $2 \mathrm{D}$ case follows from the completeness of its 1D counterpart, thanks to the one-to-one correspondence that exists between any circularly symmetric function $g(r)$ and its $1 \mathrm{D}$ cross section $p(x)$. The orthogonality property, however, is not generally guaranteed: Although in the $1 \mathrm{D}$ case the function set $\{\cos (2 \pi r n / T)\}$ is orthogonal on any interval of length $T$, this is not the case in the $2 \mathrm{D}$ circularly symmetric case, where $r$ represents $\sqrt{x^{2}+y^{2}}$, since the negative and positive volumes under the circular cosine function vary with the radius, and they do not necessarily cancel each other on a one-radialperiod interval.

As we have seen above, the main benefit of having an orthogonal set of basis functions is in the straightforward way in which the coefficients $c_{n}$ of the decomposition can be determined. However, in our case we have already succeeded, in Section 4, in determining explicitly the coefficients $c_{n}$ of the circular Fourier development-though we had to work harder.

As shown in the present paper, although the circular Fourier series may lack the property of orthogonality, it offers a useful decomposition of radially periodic images, since it clearly reveals their spectrum structure and reflects its close connection with the $1 \mathrm{D}$ spectrum of the cross section $p(x)$ of the radially periodic image.

\section{APPENDIX B: THE HALF-ORDER DERIVATIVE OF THE IMPULSE $\delta(x)$ AND ITS MAIN PROPERTIES}

Fractional-order derivatives of the impulse function $\delta(x)$ have not been widely known outside the mathematical community. However, the results presented in this paper show that half-order derivatives of the impulse function play an important role in the understanding of Fourier spectra of various circular structures. In this appendix I therefore investigate this generalized function, illustrate its behavior, and discuss its main properties.

As we have seen in Section 3, the half-order derivative of a function $f(x)$ can be expressed by

$$
f^{(1 / 2)}(x)=\mathscr{F}^{-1}\left[(i 2 \pi u)^{1 / 2} F(u)\right] .
$$

Also noted here is an elementary property of the halforder derivative that we will need later:

$$
[f(a x)]^{(1 / 2)}=\sqrt{a} f^{(1 / 2)}(a x) .
$$

An explicit expression for fractional-order derivatives of $\delta(x)$ can be found in advanced books on generalized functions, such as Ref. 13:

$$
\delta^{(\lambda)}(x)=\frac{1}{\Gamma(-\lambda)} \frac{1}{x^{\lambda+1}} \operatorname{step}(x),
$$

where $\operatorname{step}(x)$ is defined as 1 for $x>0$ and 0 for $x<0$; in particular, this gives for $\lambda=1 / 2$

$$
\delta^{(1 / 2)}(x)=-\frac{1}{2 \sqrt{\pi}} \frac{1}{x^{3 / 2}} \operatorname{step}(x)
$$

However, the best insight into the interpretation of mathematical expressions involving the impulse $\delta(x)$ is obtained by considering $\delta(x)$ as the limit of a sequence of unit-area functions. Several adequate sequences have been proposed in Ref. 25, including (i) $\tau^{-1} \operatorname{rect}(x / \tau)$, (ii) $\tau^{-1} \Lambda(x / \tau)$ and (iii) $\tau^{-1} \operatorname{sinc}(x / \tau),{ }^{26}$ all of which approach $\delta(x)$ in the limit as $\tau$ tends to zero. The question of which sequence is to be used is mainly a matter of convenience: In most cases the simplest sequence (i) will do; and only when derivatives are required, as in our case, should sequences such as (ii) or (iii) be chosen. Once the sequence to be used has been selected, the interpretation of the expression involving $\delta(x)$ is obtained by following the general three-step recipe given in Ref. 27: (1) replace 


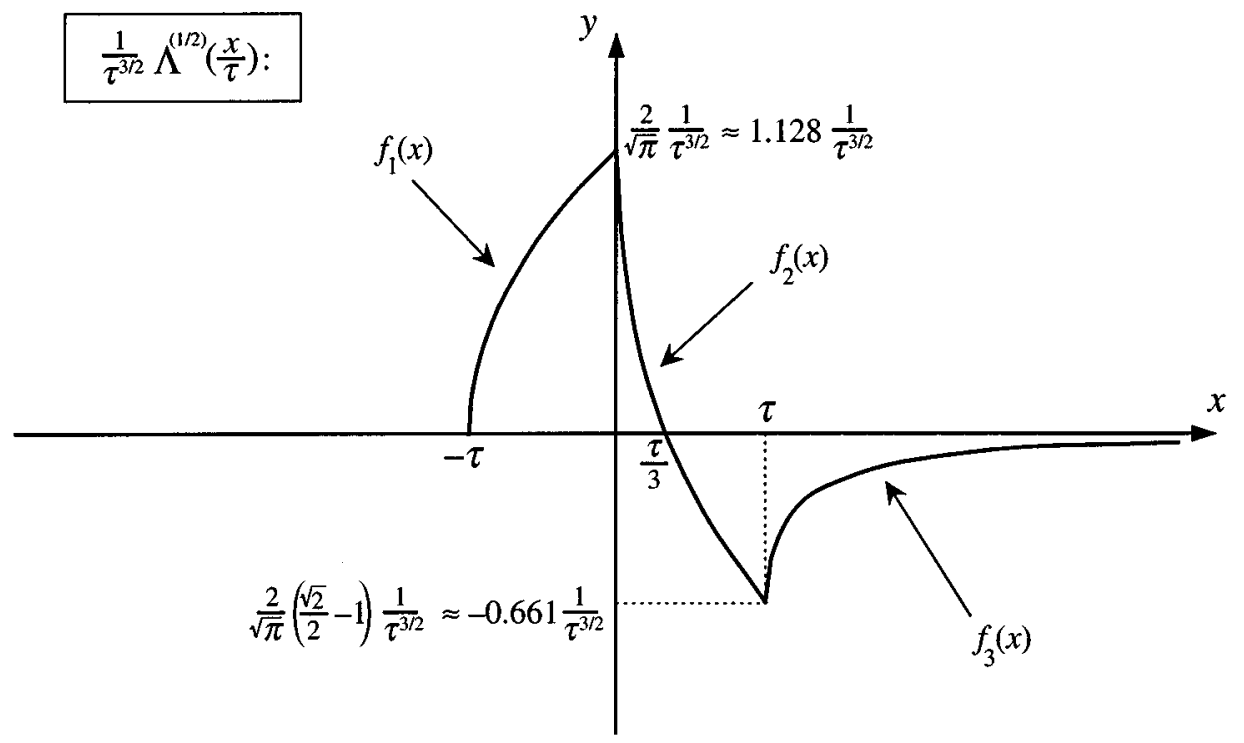

Fig. 6. Graphic representation of $\tau^{-3 / 2} \Lambda^{(1 / 2)}(x / \tau)$, the half-order derivative of $\tau^{-1} \Lambda(x / \tau)$. It is composed of three consecutive curved segments:

$$
\begin{array}{ll}
f_{1}(x)=\frac{2}{\sqrt{\pi}} \frac{1}{\tau^{3 / 2}}\left(\sqrt{\frac{x}{\tau}+1}\right) & \text { between }-\tau \leqslant x<0, \\
f_{2}(x)=\frac{2}{\sqrt{\pi}} \frac{1}{\tau^{3 / 2}}\left(\sqrt{\frac{x}{\tau}+1}-2 \sqrt{\frac{x}{\tau}}\right) & \text { between } 0 \leqslant x<\tau, \\
f_{3}(x)=\frac{2}{\sqrt{\pi}} \frac{1}{\tau^{3 / 2}}\left(\sqrt{\frac{x}{\tau}+1}-2 \sqrt{\frac{x}{\tau}}+\sqrt{\frac{x}{\tau}-1}\right) & \text { between } \tau \leqslant x<\infty
\end{array}
$$

$\delta(x)$ by the chosen sequence, (2) perform the operation in question on this sequence, and (3) proceed to the limit as $\tau \rightarrow 0$.

Therefore, to gain a better understanding of the halforder derivative of $\delta(x)$ and its properties, we follow this three-step recipe using the sequence (ii), which is the simplest sequence having a first-order derivative [note that for fractional derivatives $\delta^{(\lambda)}(x)$ of order $\lambda>1$, a sequence having more derivatives would be required]. According to this recipe, we first replace $\delta(x)$ with $\tau^{-1} \Lambda(x / \tau)$; then we perform on this sequence the operation of taking a half-order derivative; and finally, we proceed to the limit as $\tau \rightarrow 0$.

The second step of this recipe requires the calculation of the half-order derivative of $\tau^{-1} \Lambda(x / \tau)$. Since the Fourier transform of $\Lambda(x)$ is $\operatorname{sinc}^{2}(u),{ }^{28}$ we obtain from formula (B1)

$$
\begin{aligned}
\Lambda^{(1 / 2)}(x) & =\mathscr{F}^{-1}\left[(i 2 \pi u)^{1 / 2} \operatorname{sinc}^{2}(u)\right] \\
& =\int_{-\infty}^{\infty}(i 2 \pi u)^{1 / 2} \operatorname{sinc}^{2}(u) \exp (i 2 \pi x u) \mathrm{d} u .
\end{aligned}
$$

After some further manipulations, notably using $\sqrt{i}= \pm 1 / \sqrt{2} \pm i / \sqrt{2} \quad$ and $\quad \exp (i 2 \pi x u)=\cos (2 \pi x u)$ $+i \sin (2 \pi x u)$ and the mutual cancellation of integrals that appear in the calculation, we obtain

$$
\Lambda^{(1 / 2)}(x)=\frac{2}{\sqrt{\pi}}(\sqrt{x+1}-2 \sqrt{x}+\sqrt{x-1})
$$

where $\checkmark$ is considered as a real-valued function and denotes the positive value of the root. Therefore, using property (B2), we find that the half-order derivative of our sequence is

$$
\begin{aligned}
{\left[\frac{1}{\tau} \Lambda\left(\frac{x}{\tau}\right)\right]^{(1 / 2)}=} & \frac{1}{\tau^{3 / 2}} \Lambda^{(1 / 2)}\left(\frac{x}{\tau}\right) \\
= & \frac{2}{\sqrt{\pi}} \frac{1}{\tau^{3 / 2}}\left(\sqrt{\frac{x}{\tau}+1}\right. \\
& \left.-2 \sqrt{\frac{x}{\tau}}+\sqrt{\frac{x}{\tau}-1}\right) .
\end{aligned}
$$

It would be instructive to stop for a moment to examine this rather interesting result. The graphic representation of $\tau^{-3 / 2} \Lambda^{(1 / 2)}(x / \tau)$ is shown in Fig. 6. As we can see, this function consists of three distinct parts: (1) a positive, increasing branch between $-\tau \leqslant x<0$ (in which only the first square root takes part); (2) a rapidly decreasing branch between $0 \leqslant x<\tau$, which crosses the $x$ axis at $x=\tau / 3$ (in which only the first two square roots are active); and finally, (3) a negative wake that trails off between $\tau \leqslant x<\infty$ asymptotically to the $x$ axis (in which all three square roots take part).

Proceeding now to step 3 of the recipe, when $\tau \rightarrow 0$, the first branch of $\tau^{-3 / 2} \Lambda^{(1 / 2)}(x / \tau)$ tends (from the left) to an infinite positive impulse at $x=0^{-}$, and the central branch tends (from the right) to an infinite negative impulse at $x=0^{+}$. But interestingly, the wake that constitutes the third branch of $\tau^{-3 / 2} \Lambda^{(1 / 2)}(x / \tau)$ does not collapse 
onto the $x$ axis when $\tau \rightarrow 0$, but rather tends to $-1 /\left(2 \sqrt{\pi} x^{3 / 2}\right)$, which is precisely the explicit representation of $\delta^{(1 / 2)}(x)$ given in formula (B3). And indeed, it can be verified (by substituting $u=\tau / x$ and developing $\sqrt{1+u}$ and $\sqrt{1-u}$ into Taylor series) that

$$
\begin{aligned}
\lim _{\tau \rightarrow 0^{+}}\left[\frac{2}{\sqrt{\pi}} \frac{1}{\tau^{3 / 2}}\left(\sqrt{\frac{x}{\tau}+1}-2 \sqrt{\frac{x}{\tau}}+\sqrt{\frac{x}{\tau}-1}\right)\right] & \\
= & -\frac{1}{2 \sqrt{\pi}} \frac{1}{x^{3 / 2}} .
\end{aligned}
$$

Similar results can be also obtained by using the sequence $\tau^{-1} \operatorname{sinc}(x / \tau)$ rather than the sequence $\tau^{-1} \Lambda(x / \tau)$, although the calculations involved are more complicated. An explicit expression for the half-order derivative of $\operatorname{sinc}(x)$ has been derived in Appendix D of Ref. 16 and plotted there on p. 87 ; note, however, that the entity presented there is in fact the mirror image of the correct result, namely, $\operatorname{sinc} c^{(1 / 2)}(-x)$, owing to a sign inversion in the derivation (at the bottom of p. 92). In the correct representation of $\operatorname{sinc}^{(1 / 2)}(x)$, exactly as in the case of $\Lambda^{(1 / 2)}(x)$, there appear a positive peak to the left of $x$ $=0$, a negative peak to the right of $x=0$, and a negative wake that can be seen trailing off to the right, biasing the center line of the oscillations slightly downward. The graphic representation of $\tau^{-3 / 2} \operatorname{sinc}^{(1 / 2)}(x / \tau)$ is shown here in the center of Fig. 7; and indeed, taking $\tau \rightarrow 0$ gives in the limit the same characteristic behavior of $\delta^{(1 / 2)}(x)$ that we already obtained above using the simpler sequence $\tau^{-1} \Lambda(x / \tau)$.

It is interesting to note the positive impulsive behavior that we observed on the left-hand side of $\delta^{(1 / 2)}(x)$, which is clearly manifested when one is approaching $\delta^{(1 / 2)}(x)$ by

Sequence (ii): $\quad$ Sequence (iii): $\quad$ The limit as $\tau \rightarrow 0$ :

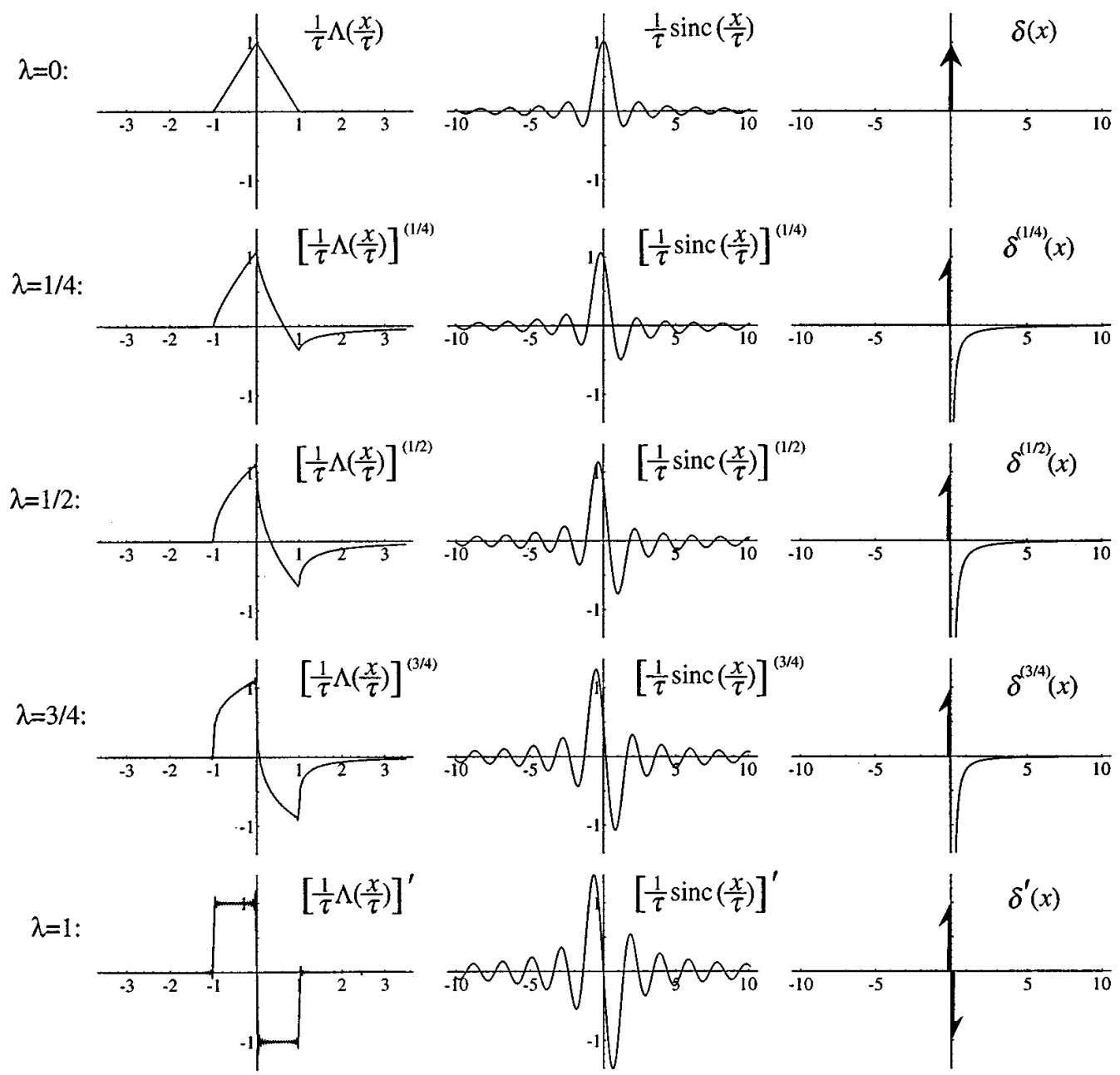

Fig. 7. Right column: graphic representations of $\delta^{(\lambda)}(x)$ for $\lambda=0,1 / 4,1 / 2,3 / 4$, and 1 . Left and middle columns: two possible sequences that approach $\delta^{(\lambda)}(x)$ in the limit as $\tau \rightarrow 0$ : the $\lambda$-order derivative of sequence (ii) (left column), and the $\lambda$-order derivative of sequence (iii) (middle column). The plots of the fractional-order derivatives of sequence (ii) and sequence (iii) were obtained by FFT, with formula (B1) (or its equivalent versions for $\lambda=1 / 4$ and 3/4). All the sequences are plotted here for $\tau=1$. Notice the gradual transition in sequences (ii) and (iii) between the plots of the derivatives of orders $\lambda=0 \ldots 1$. 
a sequence of functions that are defined on both sides of $x=0$. This impulsive behavior went unnoticed in the approach taken by Gelfand and Shilov, ${ }^{13}$ and expression (B3) that they obtain only describes the properties of $\delta^{(1 / 2)}(x)$ to the right of $x=0$. The presence of this positive peak can also be seen intuitively by considering $\delta^{(1 / 2)}(x)$ as an intermediate stage in the gradual transition between the impulse $\delta(x)$ and its derivative $\delta^{\prime}(x)$ (the inverse dipole), as shown in Fig. 7. This kind of intuitive reasoning appears also, for another case, in Ref. 29.

Finally, I give here some of the most important properties of $\delta^{(1 / 2)}(x)$, all of which can be verified by means of the three-step recipe described above:

1. Scale and shift:

$$
\begin{array}{ll}
\delta^{(1 / 2)}(a x+b)=\frac{1}{|a|^{3 / 2}} \delta^{(1 / 2)}(x+b / a) & \text { for } a>0 \\
\delta^{(1 / 2)}(a x+b)=\frac{1}{|a|^{3 / 2}} \delta^{(1 / 2)}(-x+b / a) & \text { for } a<0 .
\end{array}
$$

2. Asymmetry: In contrast to $\delta(x)$, which is symmetric [i.e., $\delta(-x)=\delta(x)$ ] and to $\delta^{\prime}(x)$, which is antisymmetric [i.e., $\delta^{\prime}(-x)=-\delta^{\prime}(x)$ ], $\delta^{(1 / 2)}(x)$ is asymmetric; $\delta^{(1 / 2)}(-x)$ is the mirror image of $\delta^{(1 / 2)}(x)$.

3. Negative wake: In addition to its impulsive behavior at $x=0, \delta^{(1 / 2)}(x)$ has a negative continuous wake that extends asymptotically in the positive direction of the $x$ axis.

4. The strength of the positive left-sided impulse (i.e., the area beneath the peak as $\tau \rightarrow 0$ ) is $\infty$; the strength of the negative right-sided impulse is $-\infty$; and even the area of the negative wake is again $-\infty$. However, the total area of $\delta^{(1 / 2)}(x)$ is 0 . This behavior (except for the wake) is reminiscent of the infinite impulse strengths of $\delta^{\prime}(x),{ }^{30}$ in contrast to $\delta(x)$, whose strength is 1 . These properties can be written symbolically as follows:

$$
\begin{gathered}
\int_{-\infty}^{\infty} \delta^{(1 / 2)}(x) \mathrm{d} x=0, \quad \int_{0}^{\infty} \delta^{(1 / 2)}(x) \mathrm{d} x=-\infty, \\
\int_{-\infty}^{\infty}\left|\delta^{(1 / 2)}(x)\right| \mathrm{d} x=\infty .
\end{gathered}
$$

5. Convolution: As a generalization of ${ }^{30}$ $\delta^{(n)}(x) * f(x)=f^{(n)}(x)$, we obtain here $\delta^{(1 / 2)}(x) * f(x)$ $=f^{(1 / 2)}(x)$

6. Fourier transform: As a generalization of $\delta^{(n)}(x) \stackrel{\mathscr{T}}{\leftrightarrow}(i 2 \pi u)^{n}$, which is received by repeated differentiation of $\delta(x) \leftrightarrow 1$, we obtain here

$$
\delta^{(1 / 2)}(x) \stackrel{\mathscr{T}}{\leftrightarrow}(i 2 \pi u)^{1 / 2} .
$$

Similar results can be also obtained for $\delta^{(\lambda)}(x)$ with any real $\lambda$; note that negative values of $\lambda$ represent $\lambda$-order integration.

\section{REFERENCES AND NOTES}

1. E. O. Brigham, The Fast Fourier Transform and Its Applications (Prentice-Hall, Englewood Cliffs, N.J., 1988), pp. 101-103, 172-173.
2. R. N. Bracewell, Two Dimensional Imaging (Prentice-Hall, Englewood Cliffs, N.J., 1995), p. 358.

3. M. Cartwright, Fourier Methods for Mathematicians, Scientists and Engineers (Ellis Horwood, Chichester, W. Sussex, UK, 1990), pp. 186-191.

4. A. Papoulis, Systems and Transforms with Applications in Optics (McGraw-Hill, New York, 1968), pp. 158-160.

5. Note that although the $n$th spectral ring is impulsive on the perimeter of a circle with radius $f=n / T$, it does not represent a pure radial frequency component of $f=n / T$, but it also includes lower radial frequencies owing its wake, which trails off toward the spectrum center.

6. R. N. Bracewell, The Fourier Transform and Its Applications, 2nd ed. (McGraw-Hill, Reading, N.Y., 1986), p. 244.

7. See, for example, J. D. Gaskill, Linear Systems, Fourier Transforms, and Optics (Wiley, New York, 1978), p. 330; or more generally for $n$ dimensions E. M. Stein and G. Weiss, Introduction to Fourier Analysis on Euclidean Spaces (Princeton U. Press, Princeton, N.J., 1971), p. 171.

8. I. M. Gelfand and G. E. Shilov, Generalized Functions (Academic, San Diego, Calif., 1964), Vol. 1, p. 185.

9. R. N. Bracewell, The Fourier Transform and Its Applications, 2nd ed. (McGraw-Hill, Reading, N.Y., 1986), p. 122.

10. S. G. Samko, A. A. Kilbas, and O. I. Marichev, Fractional Integrals and Derivatives (Gordon \& Breach, Yverdon, Switzerland, 1993).

11. K. B. Oldham and J. Spanier, The Fractional Calculus (Academic, New York, 1974).

12. R. N. Bracewell, The Fourier Transform and Its Applications, 2nd ed. (McGraw-Hill, Reading, N.Y. 1986), pp. $80-82$.

13. I. M. Gelfand and G. E. Shilov, Generalized Functions (Academic, San Diego, Calif., 1964), Vol. 1, p. 117.

14. I. M. Gelfand and G. E. Shilov, Generalized Functions (Academic, San Diego, Calif., 1964), Vol. 1, p. 237.

15. R. N. Bracewell, The Fourier Transform and Its Applications, 2nd ed. (McGraw-Hill, Reading, N.Y., 1986), p. 249.

16. R. N. Bracewell and A. R. Thompson, "The main beam and ringlobes of an east-west rotation-synthesis array," Astrophys. J. 182, 77-94 (1973).

17. R. N. Bracewell, Two Dimensional Imaging (Prentice-Hall, Englewood Cliffs, N.J., 1995), pp. 371-375.

18. R. N. Bracewell, Two Dimensional Imaging (Prentice-Hall, Englewood Cliffs, N.J., 1995), p. 374.

19. I. M. Gelfand and G. E. Shilov, Generalized Functions (Academic, San Diego, Calif., 1964), Vol. 1, p. 359.

20. R. N. Bracewell, The Fourier Transform and Its Applications, 2nd ed. (McGraw-Hill, Reading, N.Y., 1986), p. 253.

21. P. M. Morse and H. Feshbach, Methods of Theoretical Physics (McGraw-Hill, New York, 1953), Vol. 1, pp. 842-847.

22. R. S. Strichartz, A Guide to Distribution Theory and Fourier Transforms (CRC, Boca Raton, Fla., 1994), pp. 62-67.

23. J. Lighthill, Waves in Fluids (Cambridge, London, 1978), pp. 20-21.

24. J. D. Gaskill, Linear Systems, Fourier Transforms, and Optics (Wiley, New York, 1978), pp. 99-110.

25. R. N. Bracewell, The Fourier Transform and Its Applications, 2nd ed. (McGraw-Hill, Reading, N.Y., 1986), pp. $72-73$.

26. where

$$
\begin{aligned}
\operatorname{rect}(x) & = \begin{cases}1 & |x|<1 / 2 \\
0 & |x|>1 / 2\end{cases} \\
\Lambda(x) & =\left\{\begin{array}{ll}
1-|x| & |x|<1 \\
0 & |x|>1
\end{array}, \quad \operatorname{sinc}(x)=\sin (\pi x) /(\pi x) .\right.
\end{aligned}
$$

27. R. N. Bracewell, Two Dimensional Imaging (Prentice-Hall, Englewood Cliffs, N.J., 1995), p. 121.

28. R. N. Bracewell, The Fourier Transform and Its Applications, 2nd ed. (McGraw-Hill, Reading, N.Y., 1986), p. 100.

29. R. N. Bracewell, The Fourier Transform and Its Applications, 2nd ed. (McGraw-Hill, Reading, N.Y., 1986), p. 149.

30. R. N. Bracewell, The Fourier Transform and Its Applications, 2nd ed. (McGraw-Hill, Reading, N.Y., 1986), p. 82. 